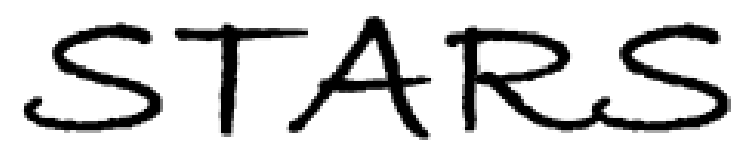

University of Central Florida

STARS

$1-1-2007$

\title{
Polarization-induced spectral changes on propagation of stochastic electromagnetic beams
}

Jixiong $\mathrm{Pu}$

Olga Korotkova

Emil Wolf

University of Central Florida

Find similar works at: https://stars.library.ucf.edu/facultybib2000

University of Central Florida Libraries http://library.ucf.edu

This Article is brought to you for free and open access by the Faculty Bibliography at STARS. It has been accepted for inclusion in Faculty Bibliography 2000 s by an authorized administrator of STARS. For more information, please contact STARS@ucf.edu.

\section{Recommended Citation}

$\mathrm{Pu}$, Jixiong; Korotkova, Olga; and Wolf, Emil, "Polarization-induced spectral changes on propagation of stochastic electromagnetic beams" (2007). Faculty Bibliography 2000s. 7538.

https://stars.library.ucf.edu/facultybib2000/7538

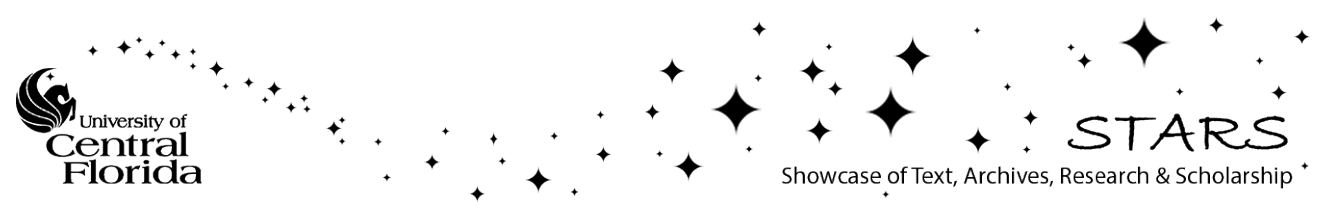




\title{
Polarization-induced spectral changes on propagation of stochastic electromagnetic beams
}

\author{
Jixiong $\mathrm{Pu}$ \\ Department of Electronic Science and Technology, Huaqiao University, Quanzhou, Fujian 362021, China \\ Olga Korotkova \\ Department of Physics and Astronomy, University of Rochester, Rochester, New York 14627, USA \\ Emil Wolf \\ Department of Physics and Astronomy, University of Rochester, Rochester, New York 14627, USA; \\ Institute of Optics, University of Rochester, Rochester, New York 14627, USA; \\ and College of Optics and Photonics: CREOL and FPCE, University of Central Florida, Orlando, Florida 32816, USA
}

(Received 17 November 2006; published 16 May 2007)

\begin{abstract}
It was shown some years ago that the spectrum of a stochastic scalar field depends not only on the source spectrum but also on the degree of coherence of the source. In this paper we show that there are electromagnetic fields for which not only the state of coherence of the source, but also its degree of polarization affect the spectrum of the radiated field. We illustrate the analysis by diagrams which show the far-zone spectra of some stochastic electromagnetic beams generated by sources of different states of coherence and different degrees of polarization. The spectra of the radiated field depend both on coherence properties of the source and its degree of polarization and are found to be different in different directions of observation.
\end{abstract}

DOI: $10.1103 /$ PhysRevE.75.056610

PACS number(s): 41.20.Jb, 42.25.Ja, 32.70.Jz, 33.70.Jg

About twenty years ago it was found that spectra of fields generated by stochastic scalar sources generally change on propagation, even in free space [1]. Many publications on this subject have appeared since then [2], but until very recently almost all investigations were based on scalar theory (see, however, [3], [4]).

In recent years there has been an increasing interest in stochastic electromagnetic sources and in the beams which they generate. A unified theory of coherence and polarization formulated several years ago [5] has provided a general description of such beams and also the laws governing their propagation [6]. More recently, stochastic beams generated by so-called electromagnetic Gaussian Schell-model sources have been studied [7-9].

Not long ago we have discussed far-zone spectra of beams generated by so-called quasihomogeneous stochastic electromagnetic sources with uniform polarization and we derived condition for the spectrum of the radiated field to be the same as the spectrum of the source [4]. In the present paper we show that there are stochastic electromagnetic sources whose degree of polarization also affects the spectra of the radiated fields.

Let us consider a planar, stochastic, statistically stationary, secondary electromagnetic source, located in the plane $z=0$, which radiates into the half space $z>0$ (Fig. 1). We assume that the source occupies a finite domain $D$ and that it generates a beam, propagating close to $z$ direction. The crossspectral density matrix of the source at a pair of points, with position vectors $\boldsymbol{\rho}_{1}=\left(x_{1}, y_{1}\right)$ and $\boldsymbol{\rho}_{2}=\left(x_{2}, y_{2}\right)$ is defined as [5]

$$
\begin{aligned}
\overleftrightarrow{W}^{(0)}\left(\boldsymbol{\rho}_{1}, \boldsymbol{\rho}_{2}, \omega\right) & =\left[W_{i j}^{(0)}\left(\boldsymbol{\rho}_{1}, \boldsymbol{\rho}_{2}, \omega\right)\right] \\
& =\left[\left\langle E_{i}^{*}\left(\boldsymbol{\rho}_{1}, \omega\right) E_{j}\left(\boldsymbol{\rho}_{2}, \omega\right)\right\rangle\right], \quad i, j=x, y .
\end{aligned}
$$

Here $E_{x}$ and $E_{y}$ are two mutually orthogonal components at frequency $\omega$ of the electric field perpendicular to the beam axis (the $z$ direction) at a typical point in the source plane and the asterisk denotes the complex conjugate. $E_{x}$ and $E_{y}$ are not Fourier components (which do not exist for statistically stationary processes) of the electric field; they must be interpreted in the sense of coherence theory in the spacefrequency domain [10]. Each of the four elements of the cross-spectral density matrix can be expressed in the form

$$
\begin{aligned}
W_{i j}^{(0)} & \left(\boldsymbol{\rho}_{1}, \boldsymbol{\rho}_{2}, \omega\right) \\
& =\sqrt{S_{i}^{(0)}\left(\boldsymbol{\rho}_{1}, \omega\right) S_{j}^{(0)}\left(\boldsymbol{\rho}_{2}, \omega\right)} \mu_{i j}^{(0)}\left(\boldsymbol{\rho}_{1}, \boldsymbol{\rho}_{2}, \omega\right), \quad i, j=x, y,
\end{aligned}
$$

where $S_{x}^{(0)}$ and $S_{y}^{(0)}$ denote the spectral densities (electric energy densities) of the $x$ and $y$ components of the electric field, in the source plane $z=0$ and $\mu_{i j}^{(0)}$ denotes the degree of correlation between the two components.

The spectral density $S^{(0)}(\boldsymbol{\rho} ; \omega)$, the spectral degree of polarization $P^{(0)}(\boldsymbol{\rho} ; \omega)$, and the spectral degree of coherence $\eta^{(0)}\left(\boldsymbol{\rho}_{1}, \boldsymbol{\rho}_{2} ; \omega\right)$ of the source are given by the formulas [5]

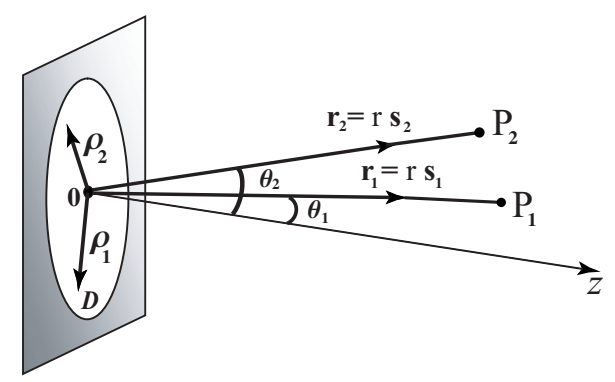

FIG. 1. (Color online) Illustrating the notation relating to propagation of beam. $\boldsymbol{\rho}_{1}$ and $\boldsymbol{\rho}_{2}$ are position vectors of points in a the source plane and $\mathbf{r}_{1}=r \mathbf{s}_{1}$ and $\mathbf{r}_{2}=r \mathbf{s}_{2}\left(\mathbf{s}_{1}^{2}=\mathbf{s}_{2}^{2}=1\right)$ are position vectors of points in the far zone. 


$$
\begin{gathered}
S^{(0)}(\boldsymbol{\rho} ; \omega)=\operatorname{Tr}\left[\overleftrightarrow{W}^{(0)}(\boldsymbol{\rho}, \boldsymbol{\rho} ; \omega)\right], \\
P^{(0)}(\boldsymbol{\rho} ; \omega)=\sqrt{1-\frac{4 \operatorname{Det}\left[\overleftrightarrow{W}^{(0)}(\boldsymbol{\rho}, \boldsymbol{\rho} ; \omega)\right]}{\left[\operatorname{Tr} \overleftrightarrow{W}^{(0)}(\boldsymbol{\rho}, \boldsymbol{\rho} ; \omega)\right]^{2}}},
\end{gathered}
$$

and

$$
\eta^{(0)}\left(\boldsymbol{\rho}_{1}, \boldsymbol{\rho}_{2} ; \omega\right)=\frac{\operatorname{Tr}\left[\overleftrightarrow{W^{(0)}}\left(\boldsymbol{\rho}_{1}, \boldsymbol{\rho}_{2} ; \omega\right)\right]}{\sqrt{S^{(0)}\left(\boldsymbol{\rho}_{1} ; \omega\right)} \sqrt{S^{(0)}\left(\boldsymbol{\rho}_{2} ; \omega\right)}},
$$

where $\operatorname{Tr}$ and Det denote the trace and the determinant of the matrix, respectively.

For simplicity we will assume that the spectral densities $S_{x}^{(0)}$ and $S_{y}^{(0)}$ are position independent, i.e., that

$$
S_{i}^{(0)}(\boldsymbol{\rho}, \omega) \equiv S_{i}^{(0)}(\omega), \quad i=x, y .
$$

Then Eqs. (3)-(5) simplify. The spectral density $S^{(0)}$ of the source becomes

$$
S^{(0)}(\boldsymbol{\rho}, \omega) \equiv S^{(0)}(\omega)=S_{x}^{(0)}(\omega)+S_{y}^{(0)}(\omega) .
$$

The normalized source spectrum may be defined by the formula

$$
S_{N}^{(0)}(\omega)=\frac{S^{(0)}(\omega)}{\int_{0}^{\infty} S^{(0)}(\omega) d \omega} .
$$

We will only consider the class of sources for which the two mutually orthogonal components $E_{x}$ and $E_{y}$ of the electric vector are uncorrelated at each point of the source, i.e., $\mu_{x y}^{(0)}(\boldsymbol{\rho}, \boldsymbol{\rho}, \omega) \equiv 0$ [11]. In this case the cross-spectral density matrix is diagonal and the expression (4) for the degree of polarization of the source reduces to

$$
P^{(0)}(\boldsymbol{\rho}, \omega)=\frac{\left|S_{x}(\omega)-S_{y}(\omega)\right|}{S_{x}(\omega)+S_{y}(\omega)} .
$$

Let us now consider the beam generated by the source. We will denote the position vectors of points in a cross section $z=z_{0}=$ const $>0$ of the beam by $\mathbf{r}$ (see Fig. 1). Expressions for the elements of the cross-spectral density matrix $\overleftrightarrow{W}$, for the spectral density $S$, for the degree of polarization $P$, for the degree of coherence $\eta$, and for the normalized spectral density $S_{N}$ are given by Eqs. (1), (3)-(5), and (8) with $\boldsymbol{\rho}_{1}$, $\boldsymbol{\rho}_{2}$, and $\boldsymbol{\rho}$ replaced by $\mathbf{r}_{1}, \mathbf{r}_{2}$, and $\mathbf{r}$, respectively.

We will now derive formulas which show how the normalized spectrum changes as the beam propagates. The elements of the cross-spectral density matrix of the electric field at a pair of points $\mathbf{r}_{1}$ and $\mathbf{r}_{2}$ in the half space $z>0$ are given by the formulas [6]

$$
\begin{aligned}
W_{i j}\left(\mathbf{r}_{1}, \mathbf{r}_{2}, \omega\right)= & \iint W_{i j}^{(0)}\left(\boldsymbol{\rho}_{1}, \boldsymbol{\rho}_{2}, \omega\right) K^{*}\left(\mathbf{r}_{1}, \boldsymbol{\rho}_{1}, \omega\right) \\
& \times K\left(\mathbf{r}_{2}, \boldsymbol{\rho}_{2}, \omega\right) d^{2} \rho_{1} d^{2} \rho_{2}, \quad i=x, y ; j=x, y,
\end{aligned}
$$

where integration extends over the source domain $D$ and

$$
K(\mathbf{r}, \boldsymbol{\rho} ; \omega)=-\frac{i k}{2 \pi z} e^{i k\left(x^{2}+y^{2}\right) / 2 z}
$$

is the paraxial wave propagation, with $k=\omega / c$. The spectral density $S(\mathbf{r}, \omega)$ of the field is given by the formula of the form of Eq. (3), viz.,

$$
S(\mathbf{r} ; \omega)=\operatorname{Tr}[\overleftrightarrow{W}(\mathbf{r}, \mathbf{r} ; \omega)]
$$

On substituting from Eq. (2) into Eqs. (10) and (12) and recalling Eq. (6) we find that

$$
S(\mathbf{r} ; \omega)=S_{x}^{(0)}(\omega) M_{x}(\mathbf{r} ; \omega)+S_{y}^{(0)}(\omega) M_{y}(\mathbf{r} ; \omega),
$$

where (no summation over repeated indexes implied)

$$
\begin{aligned}
M_{i}(\mathbf{r} ; \omega)= & \iint \mu_{i i}^{(0)}\left(\boldsymbol{\rho}_{1}, \boldsymbol{\rho}_{2} ; \omega\right) \\
& \times K^{*}\left(\mathbf{r}, \boldsymbol{\rho}_{1} ; \omega\right) K\left(\mathbf{r}, \boldsymbol{\rho}_{2} ; \omega\right) d^{2} \rho_{1} d^{2} \rho_{2}, \quad i=x, y
\end{aligned}
$$

may be called spectral modifiers of the spectral density components $S_{x}(\omega)$ and $S_{y}(\omega)$. On making use of Eq. (9) it is seen that the formula (13) for the spectral density may be expressed in terms of the degree of polarization $P^{(0)}(\omega)$ of the source as

$$
S(\mathbf{r} ; \omega)=S_{x}^{(0)}(\omega)\left[M_{x}(\mathbf{r}, \omega)+\alpha(\omega) M_{y}(\mathbf{r}, \omega)\right],
$$

where

$$
\alpha(\omega) \equiv \frac{S_{y}^{(0)}(\omega)}{S_{x}^{(0)}(\omega)}=\frac{1-P^{(0)}(\omega)}{1+P^{(0)}(\omega)},
$$

and we have assumed, without loss of generality, that $S_{x}^{(0)}(\omega) \geq S_{y}^{(0)}(\omega)$.

The normalized spectrum of the electric field at the point $\mathbf{r}$ can then be determined on substituting from Eq. (15) into the formula

$$
S_{N}(\mathbf{r} ; \omega)=\frac{S(\mathbf{r} ; \omega)}{\int_{0}^{\infty} S(\mathbf{r} ; \omega) d \omega}
$$

and one finds that

$$
S_{N}(\mathbf{r}, \omega)=\frac{S_{x}^{(0)}(\omega)\left[M_{x}(\mathbf{r}, \omega)+\alpha(\omega) M_{y}(\mathbf{r}, \omega)\right]}{\int_{0}^{\infty} S_{x}^{(0)}(\omega)\left[M_{x}(\mathbf{r}, \omega)+\alpha(\omega) M_{y}(\mathbf{r}, \omega)\right] d \omega} .
$$

We will now consider how the normalized spectrum $S_{N}$ changes when the beam propagates from the source plane to the far zone. Quantities pertaining to the far zone will be indicated by superscript $(\infty)$. The elements of the crossspectral density matrix of the electric field in the far zone, at points $\mathbf{r}_{1}=r_{1} \mathbf{s}_{1}, \mathbf{r}_{2}=r_{2} \mathbf{s}_{2}\left(\mathbf{s}_{1}^{2}=\mathbf{s}_{2}^{2}=1\right)$ are given by the expressions [Ref. [3], Eq. (3.1)] 

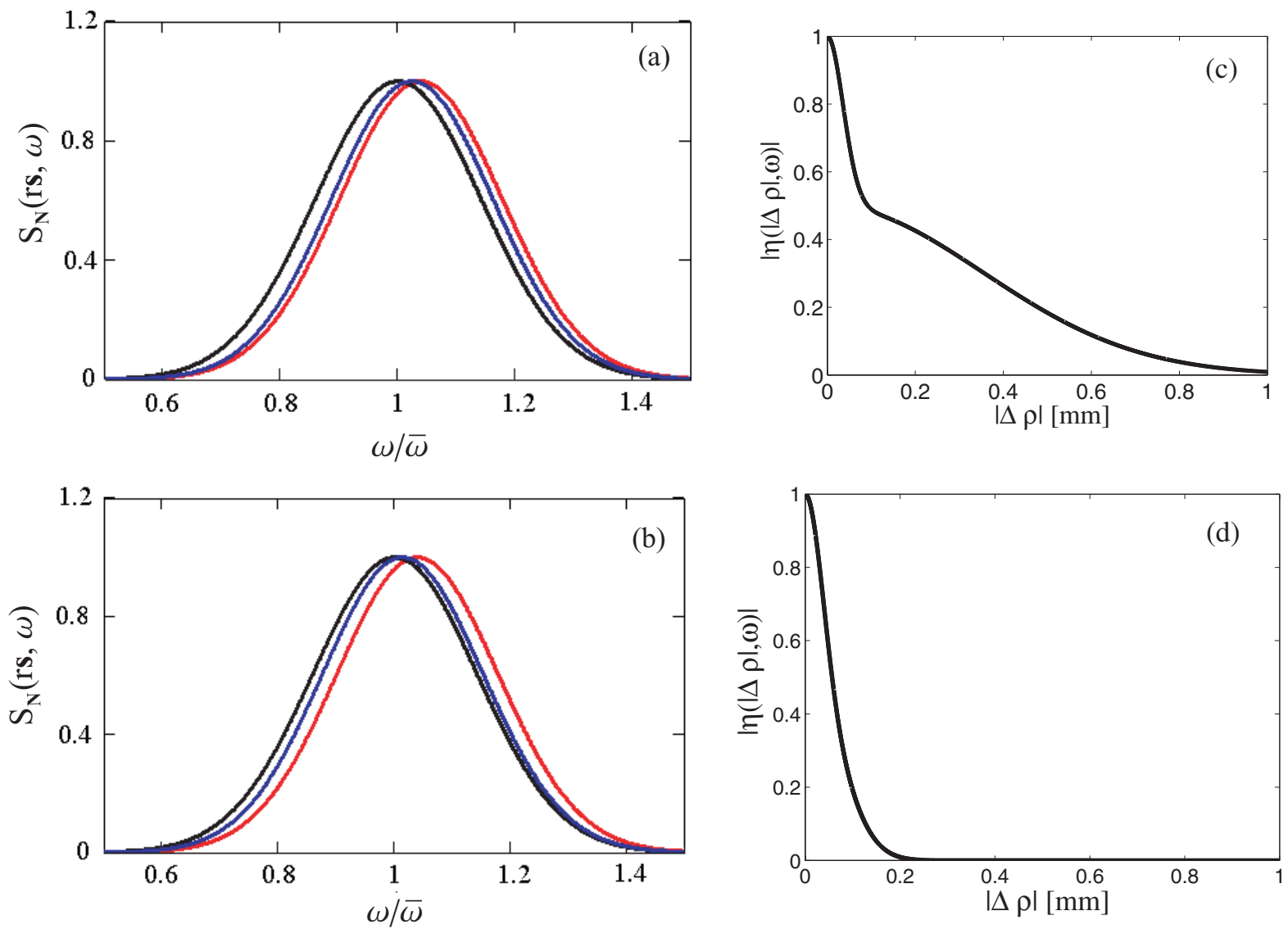

FIG. 2. (Color) (a), (b) The normalized spectral density of the far field generated by planar unpolarized $\left[P^{(0)}(\boldsymbol{\rho})=0\right]$ electromagnetic Gaussian Schell-model source, calculated from Eq. (26). The spectra are shown for different directions of observation: $\theta=0^{\circ}$, red curve; $\theta=0.3^{\circ}$, blue curve. The black curve shows the normalized spectral density of the source, calculated from Eq. (25). The parameters of the source were taken as $\sigma=0.2 \bar{\omega} / \sqrt{2}, A_{x}=1$, and $A_{y}=1, \delta_{y y}=0.05 \mathrm{~mm}$; (a) $\delta_{x x}=0.5 \mathrm{~mm}$, (b) $\delta_{x x}=0.1 \mathrm{~mm}$. (c), (d) The degree of coherence $\eta^{(0)}\left(\boldsymbol{\rho}_{1}, \boldsymbol{\rho}_{2}, \omega\right)$ of the sources in (a) and (b), respectively, for symmetrically located points, $\boldsymbol{\rho}_{2}=-\boldsymbol{\rho}_{1}=\boldsymbol{\rho}$, as function of their separation $|\Delta \boldsymbol{\rho}|=2|\boldsymbol{\rho}|$.

$$
\begin{aligned}
& W_{i j}^{(\infty)}\left(r_{1} \mathbf{s}_{1}, r_{2} \mathbf{s}_{2}, \omega\right)=4 \pi^{2} k^{2} \frac{\exp \left[i k\left(r_{2}-r_{1}\right)\right]}{r_{1} r_{2}} \\
& \quad \iint W_{i j}^{(0)}\left(\boldsymbol{\rho}_{1}, \boldsymbol{\rho}_{2}, \omega\right) \exp \left[-i k\left(\mathbf{s}_{2 \perp} \cdot \boldsymbol{\rho}_{2}-\mathbf{s}_{1 \perp} \cdot \boldsymbol{\rho}_{1}\right)\right] \\
& \quad \times d^{2} \rho_{1} d^{2} \rho_{2}, \quad i=x, y ; j=x, y,
\end{aligned}
$$

the integration extending over the source. The vectors $\mathbf{s}_{1 \perp}$ and $\mathbf{s}_{2 \perp}$ are the projections (considered as two-dimensional vectors) of the unit vectors $\mathbf{s}_{1}$ and $\mathbf{s}_{2}$ onto the source plane $z=0$ and $\theta_{1}$ and $\theta_{2}$ are the angles which $\mathbf{s}_{1}$ and $\mathbf{s}_{2}$ make with the $z$ axis (see Fig. 1). We assume that these angles are small, so that $\cos \theta_{p} \approx 1,\left|s_{p \perp}\right|^{2} \equiv \sin ^{2} \theta_{p} \approx \theta_{p}^{2}(p=1,2)$. Since the field is assumed to be beamlike, the paraxial approximation in Eq. (19) is implied.

The spectral density $S^{(\infty)}(r \mathbf{s} ; \omega)$ of the far field along the direction specified by a unit vector $\mathbf{s}$ can then be obtained from Eq. (15) with the spectral modifiers given by the formulas

$$
\begin{aligned}
M_{i}^{(\infty)}(r \mathbf{s}, \omega)= & 4 \pi^{2} k^{2} \cos ^{2} \theta \iint \mu_{i i}^{(0)}\left(\boldsymbol{\rho}_{1}, \boldsymbol{\rho}_{2}, \omega\right) \\
& \times \exp \left[-i k \mathbf{s}_{\perp} \cdot\left(\boldsymbol{\rho}_{2}-\boldsymbol{\rho}_{1}\right)\right] d^{2} \rho_{1} d^{2} \rho_{2}, \quad i=x, y
\end{aligned}
$$

The normalized spectral density of the far field can then be determined by use of Eqs. (18) and (20), viz.,

$$
\begin{aligned}
S_{N}^{(\infty)}(r \mathbf{s}, \omega) & \equiv \frac{S^{(\infty)}(r \mathbf{s}, \omega)}{\int_{0}^{\infty} S^{(\infty)}(r \mathbf{s}, \omega) d \omega} \\
& =\frac{S_{x}^{(0)}(\omega)\left[M_{x}^{(\infty)}(r \mathbf{s}, \omega)+\alpha(\omega) M_{y}^{(\infty)}(r \mathbf{s}, \omega)\right]}{\int_{0}^{\infty} S_{x}^{(0)}(\omega)\left[M_{x}^{(\infty)}(r \mathbf{s}, \omega)+\alpha(\omega) M_{y}^{(\infty)}(r \mathbf{s}, \omega)\right] d \omega},
\end{aligned}
$$

where $\alpha(\omega)$ is given by Eq. (16).

In order to illustrate spectral changes of the radiated field as the beam propagates from the source into the far zone, let 

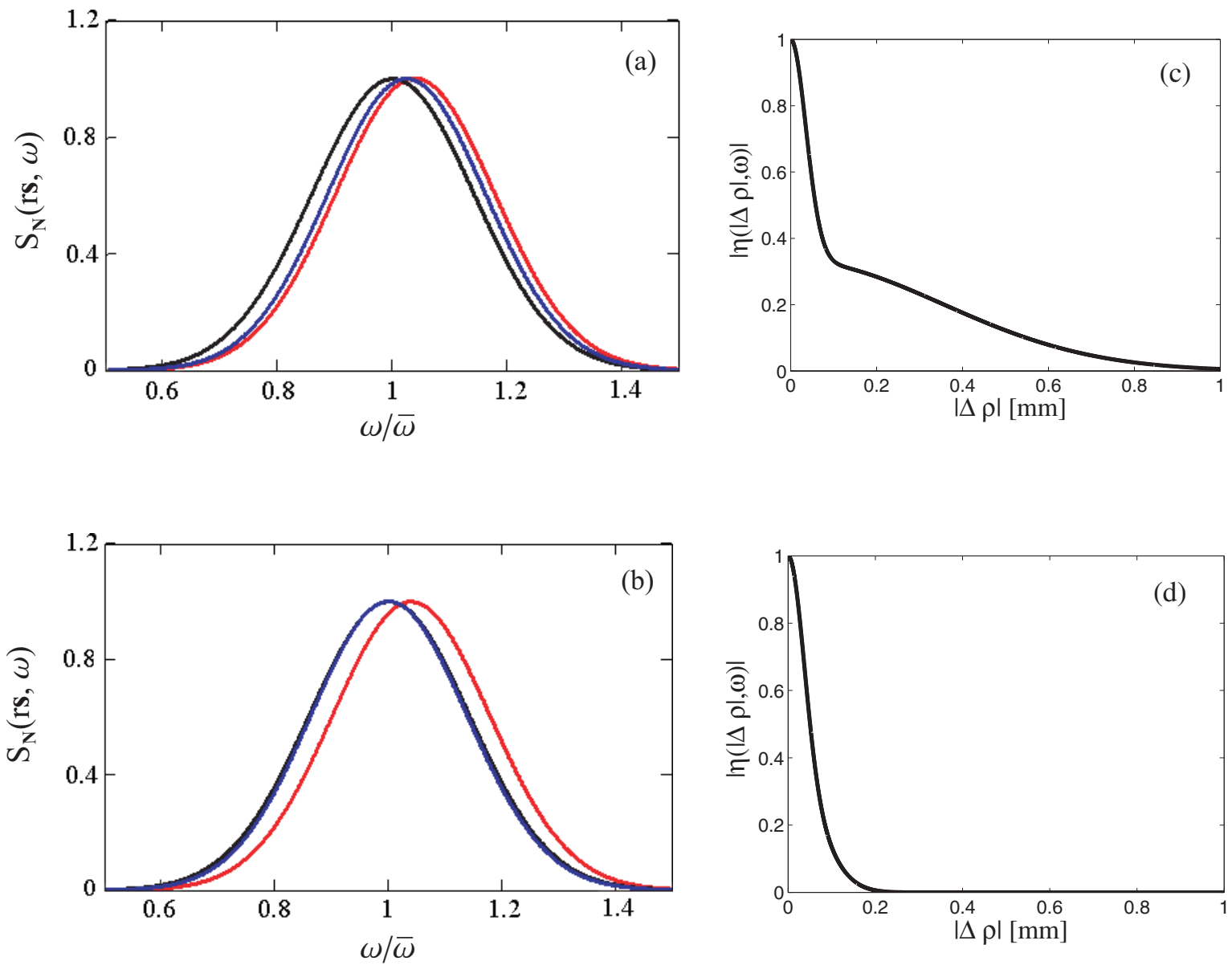

FIG. 3. (Color) The same as Fig. 2 but for a partially polarized source with degree of polarization $P^{(0)}(\boldsymbol{\rho})=1 / 3$. The parameters of the source were chosen to be $A_{x}=1$ and $A_{y}=2$.

us consider a planar, secondary electromagnetic Gaussian Schell-model source $[3,7,9]$, with uncorrelated field components $E_{x}$ and $E_{y}\left[\right.$ i.e. with $\left.\mu_{i j}^{(0)}\left(\boldsymbol{\rho}_{1}, \boldsymbol{\rho}_{2} ; \omega\right)=0\right]$ in the chosen set of $x, y$ axes. The elements of the cross-spectral density matrix of such a source have the form (no summation over repeated indexes is implied)

$$
\begin{aligned}
& W_{i j}^{(0)}\left(\boldsymbol{\rho}_{1}, \boldsymbol{\rho}_{2} ; \omega\right) \\
& \quad=S_{i}^{(0)}(\boldsymbol{\rho}, \omega) \mu_{i i}^{(0)}\left(\boldsymbol{\rho}_{2}-\boldsymbol{\rho}_{1}, \omega\right), \quad \text { if } i=j, \\
& \quad=0, \quad \text { if } i \neq j,
\end{aligned}
$$

where $S_{x}^{(0)}(\boldsymbol{\rho}, \omega)$ and $S_{y}^{(0)}(\boldsymbol{\rho}, \omega)$ are the spectra of the electric field components $E_{x}$ and $E_{y}$, in the source plane, and $\mu_{i i}^{(0)}\left(\boldsymbol{\rho}_{2}-\boldsymbol{\rho}_{1}, \omega\right)$ represents the degrees of correlation of the components $E_{i}$ at points $\boldsymbol{\rho}_{1}$ and $\boldsymbol{\rho}_{2}$ in that plane. We further assume that the spectra $S_{x}^{(0)}$ and $S_{y}^{(0)}$ are position independent and have Gaussian spectral profiles, i.e., that

$$
S_{i}^{(0)}(\boldsymbol{\rho}, \omega)=A_{i}^{2} \exp \left[-\frac{(\omega-\bar{\omega})^{2}}{2 \sigma^{2}}\right]
$$

and that the linear dimensions of the source are large compared to $\sigma$. The quantities $A_{i}$ and $\sigma^{2}$ are assumed to be independent of position and of frequency. For the sake of sim- plicity we have assumed that the variances of the spectra of the $x$ and the $y$ components are the same.

Suppose that the correlation coefficients $\mu_{i i}^{(0)}$ also have Gaussian form with respect to their spatial arguments, i.e., that

$$
\mu_{i i}^{(0)}\left(\boldsymbol{\rho}_{2}-\boldsymbol{\rho}_{1}, \omega\right)=\exp \left[-\frac{\left(\boldsymbol{\rho}_{2}-\boldsymbol{\rho}_{1}\right)^{2}}{2 \delta_{i i}^{2}}\right], \quad i=x, y,
$$

where the parameters $\delta_{i i}$ are assumed to be independent of position.

On substituting from Eqs. (22)-(24) into Eq. (8) one finds that the normalized source spectrum is given by the expression

$$
S_{N}^{(0)}(\boldsymbol{\rho}, \omega)=\frac{1}{\sigma} \sqrt{\frac{2}{\pi}} \frac{\exp \left[-\frac{(\omega-\bar{\omega})^{2}}{2 \sigma^{2}}\right]}{1+\operatorname{erf}(\bar{\omega} / \sigma \sqrt{2})},
$$

where erf denotes the error function.

An expression for the normalized spectral density of the far field can be readily obtained by first substituting from Eq. (24) for $\mu_{i i}$ into Eq. (20) and then by substituting the resulting expression (with $\mathbf{r}_{1} \mathbf{s}_{1}=\mathbf{r}_{2} \mathbf{s}_{2}=\mathbf{r s}$, and also the right-hand side of Eq. (23) into formula (21b). One then finds, after long but straightforward calculations, that 

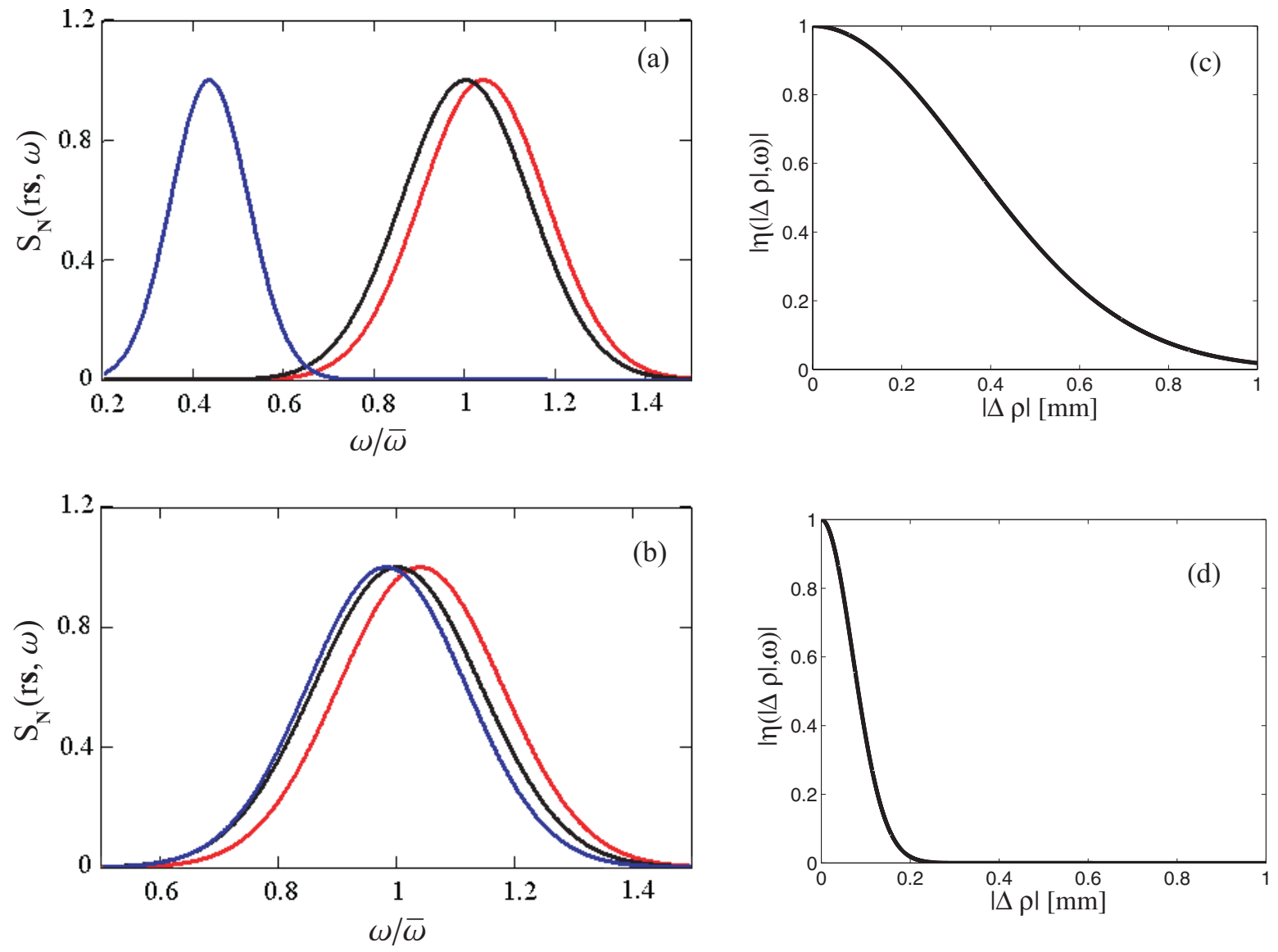

FIG. 4. (Color) The same as Fig. 2 but for a fully polarized source $\left[P^{(0)}(\boldsymbol{\rho})=1\right]$, with $A_{x}=1$ and $A_{y}=0$.

$$
S_{N}^{(\infty)}(r \mathbf{s}, \omega)=\frac{f(r \mathbf{s}, \omega)}{g(r \mathbf{s}, \omega)},
$$

where

$$
\begin{gathered}
f(r \mathbf{s}, \omega)=A_{x}^{2} \omega^{2} \exp \left[-\frac{(\omega-\bar{\omega})^{2}}{2 \sigma^{2}}\right]\left\{\delta_{x x}^{2} \exp \left[-\frac{\omega^{2}}{K_{x}^{2}}\right]\right. \\
\left.+\alpha(\omega) \delta_{y y}^{2} \exp \left[-\frac{\omega^{2}}{K_{y}^{2}}\right]\right\} \\
g(r \mathbf{s})=\int_{0}^{\infty} f(r \mathbf{s}, \omega) d \omega
\end{gathered}
$$

and

$$
K_{i}^{2}=\frac{2 c^{2}}{\theta^{2} \delta_{i i}^{2}}, \quad i=x, y
$$

The integrals entering in Eq. (28) can be expressed in closed form. One finds with the help of MATHEMATICA that

$$
\begin{aligned}
\int_{0}^{\infty} \omega^{2} \exp \left[-\frac{(\omega-\bar{\omega})^{2}}{2 \sigma^{2}}\right] \exp \left[-\frac{\omega^{2}}{K_{i}^{2}}\right] d \omega \\
=\frac{1}{4 c_{1} c_{2}^{2}}\left\{\operatorname { e x p } ( - \frac { \overline { \omega } } { 2 \sigma ^ { 2 } } ) K _ { i } ^ { 2 } \left[4 c_{3} \sqrt{\pi} \sigma^{4}+2 K_{i}^{2} c_{3} \sqrt{\pi} \sigma^{2}\right.\right. \\
+4 c_{1} \sigma^{2} \bar{\omega}+2 c_{3} \sqrt{\pi} \bar{\omega}^{2}+c_{3} \sqrt{\pi}\left[4 \sigma^{4}\right. \\
\left.\left.\left.+2 K_{i}^{2}\left(\sigma^{2}+\bar{\omega}^{2}\right)\right] \operatorname{erf}\left[\frac{K_{i}^{2} c_{1} \bar{\omega}}{c_{i}^{2}}\right]\right]\right\}, \quad i=x, y,
\end{aligned}
$$

where

$$
\begin{gathered}
c_{1}=\sqrt{\frac{1}{K_{x}^{2}}+\frac{1}{2 \sigma^{2}}}, \quad c_{2}=K_{x}^{2}+2 \sigma^{2}, \\
c_{3}=\exp \left[\frac{K_{x}^{2} \bar{\omega}^{2}}{2 K_{x}^{2} \sigma^{2}+4 \sigma^{4}}\right] .
\end{gathered}
$$

We will now use formulas (26)-(28) to illustrate how the degree of polarization, and the degree of coherence of the source affect the spectrum of the far field in some typical cases. Figures 2-4 show the spectral densities of the far field generated by an unpolarized source $\left(P^{(0)}=0\right)$, by a partially polarized source, with $P^{(0)}=1 / 3$, and by a completely polarized $\left(P^{(0)}=1\right)$ planar, electromagnetic, Gaussian Schellmodel sources calculated by use of Eq. (26). Curves illustrat- 
ing the behavior of the degrees of coherence of the three sources are also shown in the figures. Although some of the spectral changes are rather small, they are well within the capability of experimental verification.

We conclude by saying we have shown that the spectrum of the field in the far zone, radiated by some stochastic, statistically stationary planar electromagnetic sources may depend both on the degree of coherence and on the degree of polarization of the source. We illustrated this phenomenon by several curves, which also indicate the changes in the spectra of the far field in different directions of observation.

This research was supported by the U.S. Air Force Office of Scientific Research under Grant No. FA 9550-06-1-0032, by the Air Force Research Laboratory (AFRC) under Contract No. FA 9451-04-C-0296, and by the National Natural Science Foundation of China under Grant No. 60477041.
[1] E. Wolf, Phys. Rev. Lett. 56, 1370 (1986).

[2] For review of many publications on this subject see E. Wolf and D. F. V. James, Rep. Prog. Phys. 59, 771 (1996).

[3] O. Korotkova, B. Hoover, V. Gamiz, and E. Wolf, J. Opt. Soc. Am. A 22(11), 2547 (2005).

[4] J. Pu, O. Korotkova, and E. Wolf, Opt. Lett. 31, 2097 (2006).

[5] E. Wolf, Phys. Lett. A 312, 263 (2003).

[6] E. Wolf, Opt. Lett. 28, 1078 (2003).

[7] F. Gori, M. Santarsiero, G. Piquero, R. Borghi, A. Mondello, and R. Simon, J. Opt. A, Pure Appl. Opt. 3, 1 (2001).

[8] O. Korotkova, M. Salem, and E. Wolf, Opt. Lett. 29, 1173 (2004).

[9] T. Shirai, O. Korotkova, and E. Wolf, J. Opt. A, Pure Appl. Opt. 7, 232 (2005).
[10] L. Mandel and E. Wolf, Optical Coherence and Quantum Optics (Cambridge University Press, Cambridge, 1995), Sec. 4.7.

[11] The condition $\mu_{x y}^{(0)}(\boldsymbol{\rho}, \boldsymbol{\rho}, \omega) \equiv 0$ depends, of course, on the choice of the $x, y$ axes. When it is satisfied the axes are along the eigenvectors of the matrix $\overleftrightarrow{W}^{(0)}(\boldsymbol{\rho}, \boldsymbol{\rho}, \omega)$ and the spectra $S_{x}(\boldsymbol{\rho}, \omega)$ and $S_{y}(\boldsymbol{\rho}, \omega)$ are the eigenvalues of the matrix. Although the matrix can always be diagonalized by a unitary transformation, the transformation may not be a pure rotation. Physically this implies that, in general, one would need not only rotation but also a phase delay to obtain a transformed field for which the degree of polarization is given by the formula (9). For a fuller discussion of this point, see A. AlQasimi, O. Korotkova, D. James, and E. Wolf, Opt. Lett. 32, 1015 (2007). 